\title{
Estudio de generación y composición de residuos sólidos en el cantón de Guácimo, Costa Rica
}

\author{
Solid waste generation and composition \\ study in Guácimo, Costa Rica
}

Rooel Campos-Rodríguez'

Silvia Soto-Córdoba

Fecha de recepción: 06 de diciembre del 2013

Fecha de aprobación: 18 de marzo del 2014

2 Doctora en Ciencias Ambientales. Catedrática, Escuela de Ingeniería Ambiental, ITCR. Correo electrónico ssoto@itcr.ac.cr. 


\section{Palabras clave}

Residuos sólidos; estudios de composición; municipalidad de Guácimo; índice de generación de residuos sólidos.

\section{Resumen}

La Municipalidad de Guácimo, en la provincia de Limón, no cuenta con un estudio de composición ni tiene establecidos los índices de generación de desechos sólidos por habitante y comercios, por lo cual no ha logrado planificar adecuadamente sus actividades en este campo. En esta investigación se determinaron los índices de generación de desechos sólidos por habitante y su composición porcentual según los tipos de materiales contemplados en la legislación costarricense; además se brindan sugerencias para diseñar un Plan de Gestión Integral de Residuos Sólidos.

Para esto se seleccionaron los barrios por estudiar según sus ingresos económicos de acuerdo con el reglamento de estudios de composición del país, y se aplicaron herramientas estadísticas para determinar los índices de generación según el estrato socioeconómico, además de cuantificar porcentualmente los tipos de materiales que se generaban en el cantón. Los datos resultantes sirven de base para una oportuna planificación de las inversiones, establecer rutas de recolección según las verdaderas necesidades comunitarias, identificar opciones de mejora, mejorar el porcentaje de cobertura -que en estos momentos ronda el 65\%-, identificar las acciones de educación ambiental tendientes a la reducción y separación en la fuente, apoyar la creación de centros de transferencia de materiales y diseñar estrategias para la recolección selectiva.

Para el sector urbano se determinó un índice de generación promedio de 0,55 \pm 0,1 kg/persona/día y una distribución porcentual de los residuos sólidos del orden del 35\% de materiales valorizables, $45 \%$ de residuos biodegradables y $20 \%$ de materiales para vertido en relleno sanitario; mientras que en el sector comercial los porcentajes de materiales valorizables fueron del $65 \%$ y para los materiales biodegradables rondó el $15 \%$ y un $20 \%$ de material de vertido.

Los autores esperan que esta información permita utilizar en forma racional y sostenible los recursos económicos y de personal de que dispone la Municipalidad y con esto lograr mejorar la situación ambiental de la zona.

\section{Key words}

Solid waste; composition and generation rates; per capita studies; Municipalidad de Guácimo.

\begin{abstract}
The Guacimo municipality is located in the province of Limón. They do not have composition and generation rates per capita studies, because of that they can not to plain adequacy their activities. We determinate the generation rates per capita and the percentage composition of solid waste materials according the Costa Rican law.
\end{abstract}

We use the document of composition and generation studies, the data was analyzed using statistics, we determinate the generation index according the social economic status, and quantify the different kinds of materials that was produces in the place.

The results are useful for the investment planning, elaborates the collection routes according the real communitarian necessities, identify the better options for improve their environmental actions, improve the cover percentage because in this moments its around $65 \%$, identify the environmental education actions for decrease the generation of solid waste, and promotes selective solid waste classifies from houses, promotes the creation of recycle centers and design the strategies for selective solid waste collection.

We find for urban sector generation an index averaged 0,55 $\pm 0,1 \mathrm{~kg} /$ person / day, the percentage distribution of solid waste was $35 \%$ of potential recycle materials, $45 \%$ biodegradable and $20 \%$ of materials for disposition in landfill. While the commercial sector, the potential recycle materials were $65 \%$, for biodegradable materials $15 \%$ and $20 \%$ of dumped material.

The authors pretend that this information allows, the rational and sustainable economic use for determine the personnel and the resources of the municipality, for improve the environmental situation in the area. 


\section{Introducción}

Los estudios de composición de materiales son una herramienta valiosa para la planificación de actividades en los gobiernos municipales, ya que determinan el índice de generación por habitante (ppc) y la composición porcentual de los materiales que son desechados. El uso de estos indicadores y los porcentajes de contribución de cada tipo de material que se desecha permiten estimar las inversiones necesarias para dedicarlas a educación ambiental, transporte, disposición, tratamiento y administración y, por ende, hacer cálculos realistas de los costos del servicio. Además, le indica al municipio los costos en inversiones en caso de que se quiera invertir en centros de recuperación de materiales, compostaje de material biodegradable o cualquier otra opción alternativa al tratamiento de vertidos en rellenos sanitarios.

En el caso de la Municipalidad de Guácimo, específicamente en la comunidad de Pocora, se habían realizado algunos sondeos para determinar la composición y el índice de generación (Dicent, Yeoman \& Argüedas, 2008; Camacho, Murillo \& Yeomans, 2008; Abubacar, Acosta \& Yeomans, 2008). Sin embargo, se evidenció que se requería una muestra más robusta y el posterior análisis estadístico más riguroso de los datos, de forma que pudieran servir para predecir y planificar la gestión integral de los residuos sólidos (RS) en el cantón.

La Municipalidad de Guácimo cuenta con 1 1,797 viviendas ocupadas con un promedio de 3,5 habitantes por unidad habitacional (INEC, 20II). El servicio de recolección se le brinda al 65\% de las viviendas. La forma en que se disponen y se manejan los RS en el cantón ha sido ampliamente discutida por Campos \& Soto (20।4), evidenciándose la contaminación de ríos, lotes y caños por los deficientes sistemas de recolección de basura, y del suelo donde se ubica el botadero a causa del vertido incontrolado.

En Costa Rica, a partir del año 2010 se hizo obligatorio el manejo adecuado de los RS, y en 2012 se publicó el primer reglamento para realizar los respectivos estudios de composición. Actualmente es obligatorio para todos los cantones contar con estos estudios de generación y composición de RS.
Este reglamento tiende a establecer un método general para hacer los estudios de composición, sin embargo, los autores consideran que es necesario realizar una mejor y más robusta interpretación estadística de los resultados, a fin de poder estimar y predecir las cantidades generadas en el cantón.

Guácimo es el sexto cantón de la provincia de Limón, Costa Rica. Las coordenadas geográficas

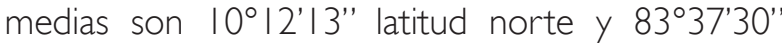
longitud oeste (figura I), su territorio abarca 576.48 kilómetros cuadrados y su población es de 41,266 habitantes, según el Instituto Nacional de Estadística y Censos (INEC, 20 I I).

El cantón está dividido en cinco distritos: Guácimo, Mercedes, Pocora, Río Jiménez y Duacarí. Los límites son: al norte y al oeste el cantón de Pococí, al sur el cantón de Turrialba, provincia de Cartago, y al este Siquirres (PNUD, FOMUDE, 2009).

El cantón de Guácimo presenta un Índice de Desarrollo Social (IDS) de 47,8 que es uno de los más bajos del país, y además se encuentra en la provincia con mayor rezago social y económico, con problemas de pobreza y deterioro de los ecosistemas y los servicios públicos (FOMUDE, 2008).

\section{Metodología}

Área de estudio

El estudio se desarrolló en la zona urbana del cantón de Guácimo, como se muestra en las figuras 2 y 3.

Criterios de selección de la muestra

Para realizar el estudio de composición se consideró el criterio de los encargados municipales, se analizó la estructura de distribución de la población en el cantón y se identificaron los sitios de mayor acceso.

Se determinó que el 73\% de la población se concentra en el centro urbano de Guácimo (distritos de Guácimo y Jiménez) y solo a estos sectores se les brinda el servicio de recolección municipal de desechos. El resto de la población del cantón no recibe el servicio de recolección pues se encuentra distribuida en zonas rurales poco densificadas. Por esta razón, este estudio se concentró en el grupo que recibe recolección municipal, que a su vez coincidentemente corresponde al más densificado. 


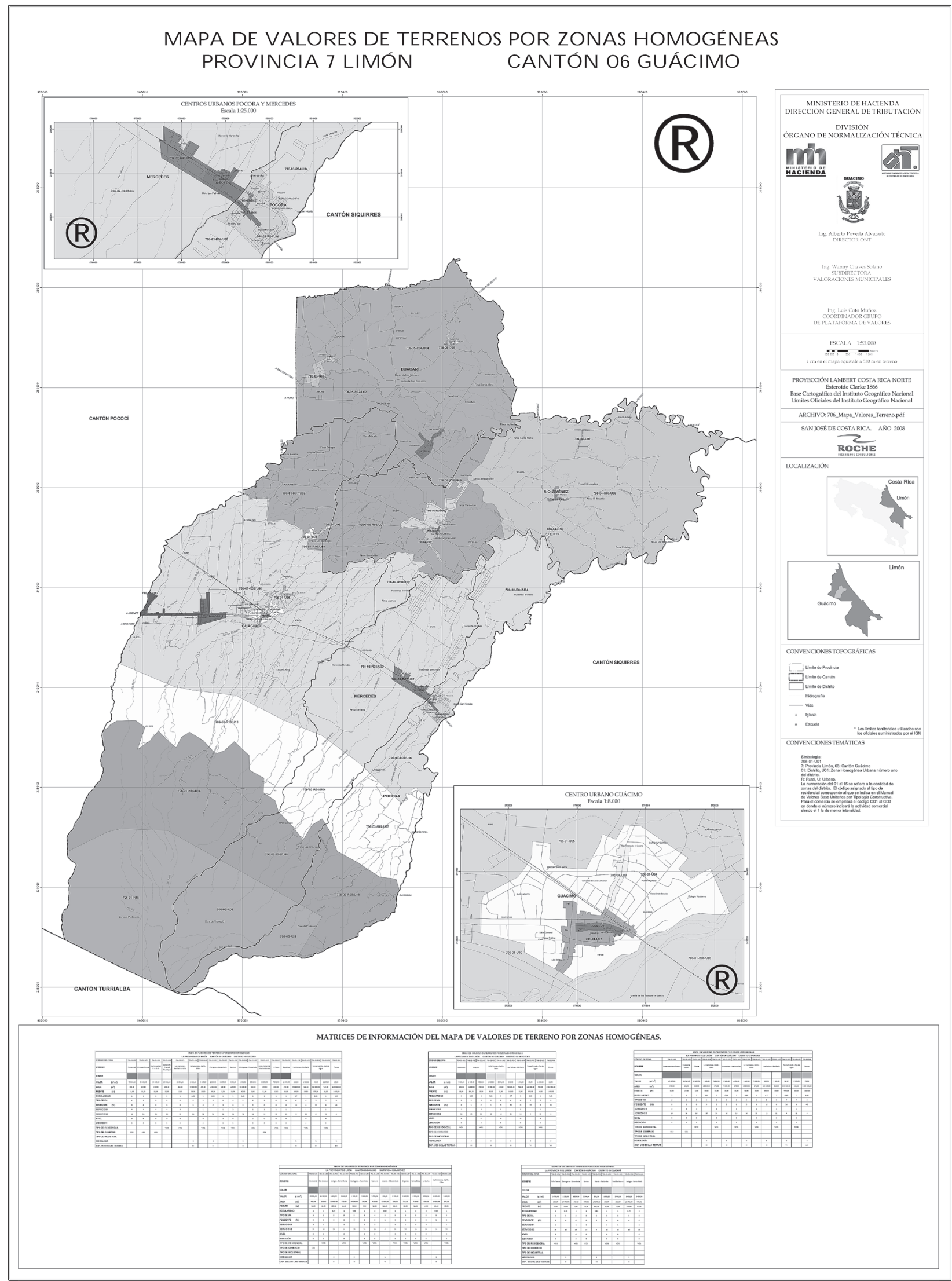

Figura I. Mapa del cantón de Guácimo. 


\section{Determinación y cálculo de la}

muestra del sector vivienda

Según el Reglamento de estudios de composición y generación de residuos sólidos, se deben identificar y seleccionar tres estratos socioeconómicos para el sector residencial, a saber: Estrato Alto (EA), Estrato Medio (EM) y Estrato Bajo (EB).

Para definir los estratos sociales, debido a que la zona no cuenta con esta información, se recurrió al INEC, se consultó el mapa de valores de terrenos por zonas homogéneas del Ministerio de Hacienda y se hizo una aproximación utilizando resultados de quintiles de ingreso per cápita de la zona Huetar Atlántica, pues su perfil socioeconómico es similar al del cantón de Guácimo. Posteriormente, se consultó con los encargados municipales y el Ministerio de Salud, que corroboraron los límites establecidos para cada estrato socioeconómico. Para el cantón de Guácimo se definieron los siguientes límites: Estrato Bajo, hasta un ingreso de US\$596; Estrato Medio, de U\$597 a US\$1,217; Estrato Alto, de US\$1,21 8 en adelante. El muestreo se realizó en los distritos de Guácimo y Jiménez, dado que la mayoría de la actividad comercial y poblacional se concentra en ellos.

En reuniones con la Municipalidad, el Ministerio de Salud, y utilizando los criterios del INEC, se identificaron las zonas o barriadas donde se agrupaban las poblaciones que se podrían categorizar en cada uno de los estratos, por lo que se aprovechó esta característica para diseñar una estructura de muestreo en forma de bloques geográficos, que se muestran en las figuras 2 y 3 . Estas zonas corresponden a tres barrios representativos según cada estrato socioeconómico. El barrio La Clínica, ubicado en latitud $10^{\circ} 12^{\prime} 40^{\prime \prime} \mathrm{N}$, longitud 834 40' 89" O, corresponde a la clase alta de la zona; por su parte, el barrio Los Geranios, latitud $10^{\circ} 12^{\prime} 46^{\prime \prime} \mathrm{N}$, longitud 83० 4I' I2" $\mathrm{O}$, corresponde a la clase media; y finalmente en el distrito de Río Jiménez se muestreó la población del Barrio de Santa María, latitud $10^{\circ}$ I5' 82" N, longitud $83^{\circ} 36^{\prime} 64^{\prime \prime} \mathrm{O}$, que corresponde a la clase baja.

La muestra seleccionada ascendió a I7I viviendas, 27 del estrato alto (que corresponde al 15\% de la población de Guácimo), 66 del estrato medio (39\% de la población) y 78 del estrato bajo (46\% de la población), calculados con la siguiente fórmula:

$$
n=\frac{(Z 2 * N * \delta 2)}{(N-1) * E 2+(Z 2 * \delta 2)} * 1.25 \ldots
$$

Fórmula I. Cálculo de muestra

Donde:

n: número de viviendas que incluirá la muestra

N: número total de viviendas del cantón

$Z$ : coeficiente de confianza al 95\%=1,96

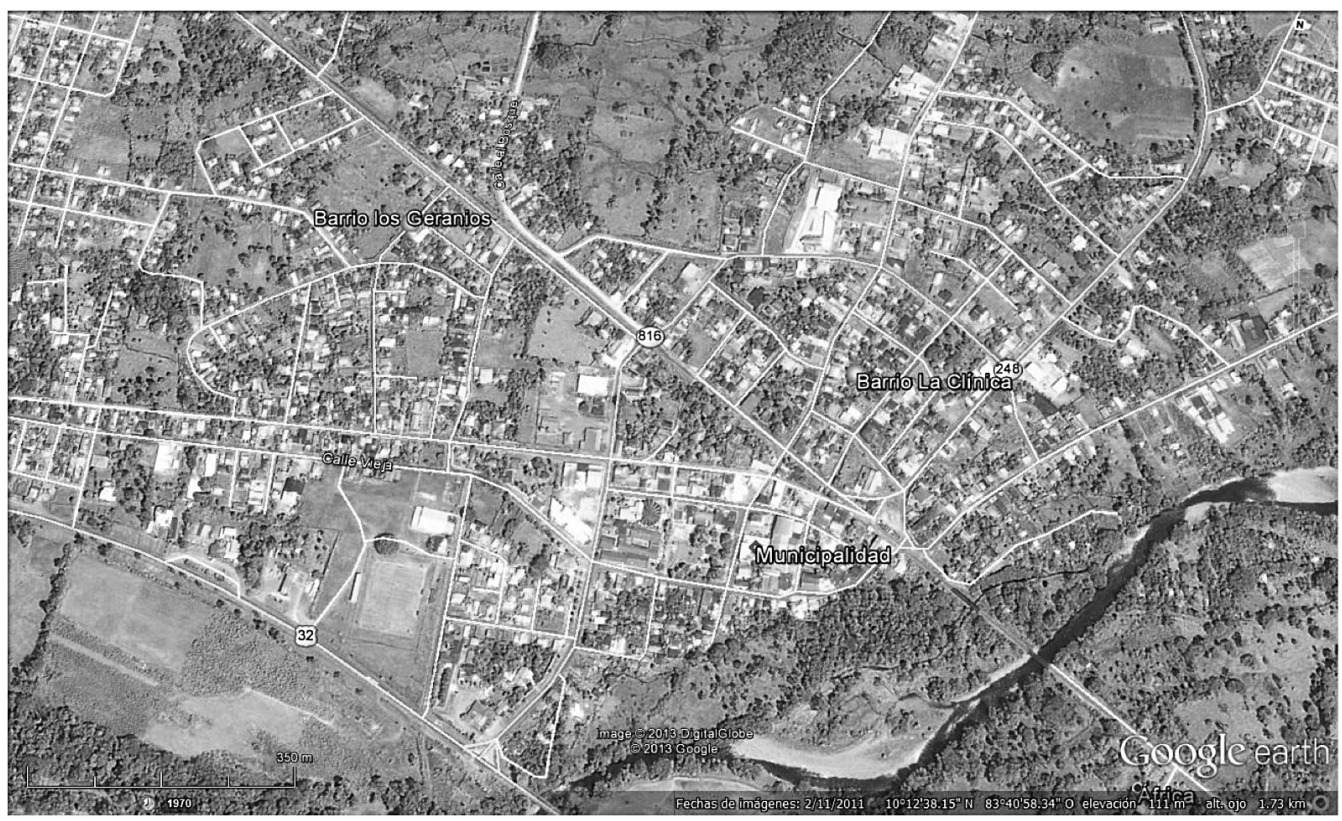

Figura 2.Vista satelital de los barrios La Clínica y Los Geranios 


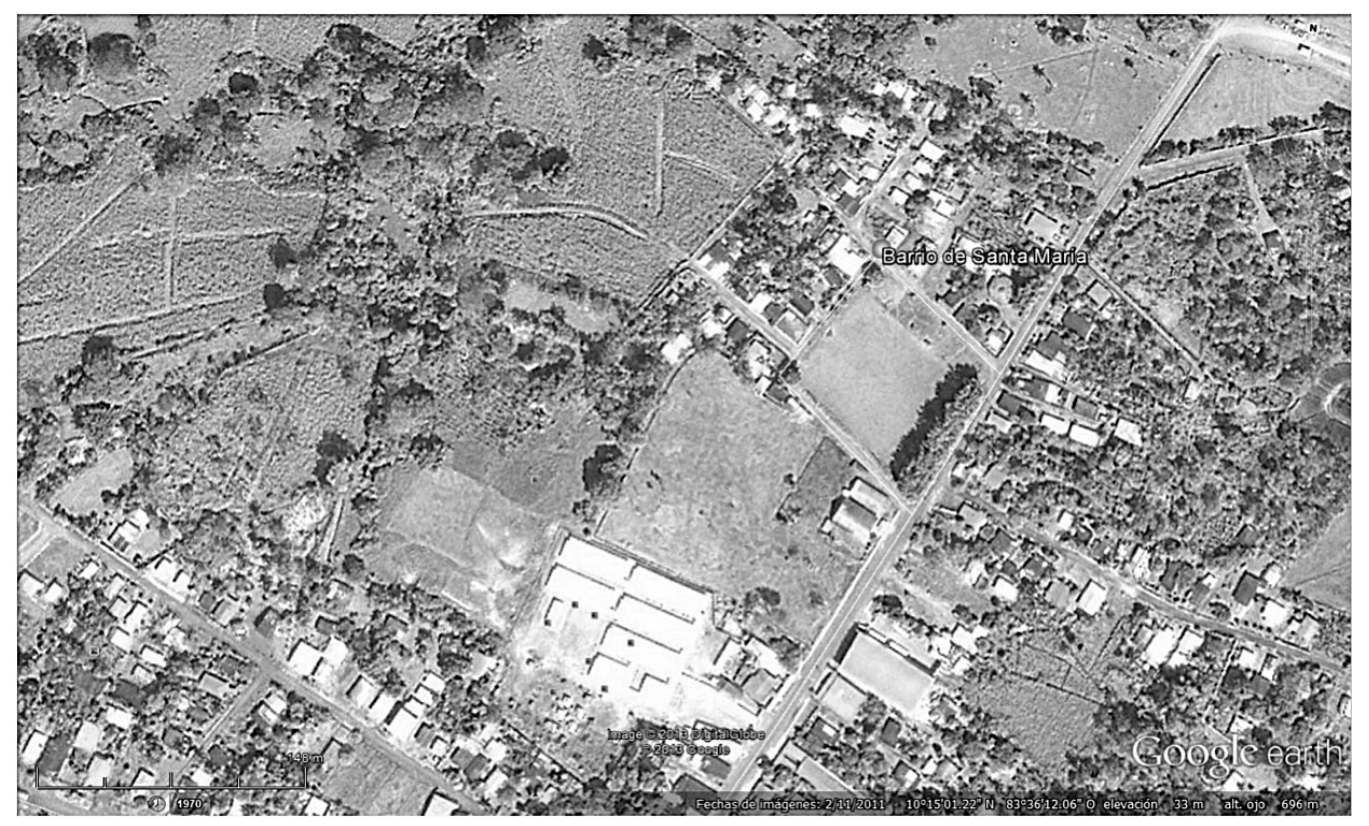

Figura 3. Vista satelital del Barrio Santamaría.

$\delta$ : desviación estándar $=0,3 \mathrm{~kg} /$ habitante $/$ día

E: error permisible $=0,05 \mathrm{~kg} /$ habitante $/ \mathrm{dí}$

\section{Determinación y cálculo de la} muestra del sector comercial

En el sector comercial se clasificaron las actividades según el Código Industrial Internacional Uniforme (CIIU). En Guácimo existen 670 comercios (cuadro I), de los cuales se definió una muestra representativa con 52 comercios al azar, para esto se utilizó la fórmula I, con una desviación estándar de 0,5 $\mathrm{kg} / \mathrm{habitante} / \mathrm{d}$ á y un error permisible de $0,15 \mathrm{~kg} /$ habitante/día.

Además, con el objetivo de investigar si existía alguna variación en los índices de generación y en la composición de los RS según el tipo de actividad comercial, se decidió analizar otra segunda muestra representativa, que correspondió al sector de los supermercados, considerando que es el que tiene el mayor peso porcentual dentro del grupo de comercios.

\section{Toma de muestras}

Antes de la toma de muestras se hizo una encuesta con el propósito de definir la percepción y sensibilización en torno al tema de los RS. Esta encuesta también sirvió de base para identificar el número de personas por vivienda y terminar de clasificar el estrato socioeconómico. Para aplicar la encuesta, se comenzó la entrevista desde la primera casa ubicada en la entrada de cada barrio hasta terminar el número de encuestas que se debían realizar por estrato. A las personas que participaron en la encuesta se les solicitó su permiso para colocar un adhesivo con un número consecutivo en la puerta de sus casas, con el fin de identificar la vivienda para la posterior toma de muestra del estudio de generación y composición.

Debido a que la recolección de residuos sólidos en Guácimo se realiza una vez por semana, los residuos que se recolectaron correspondieron a una generación semanal. El estudio se desarrolló desde el 23 de mayo hasta el 7 de junio de 2013.

Determinación de la composición de los RS y del índice de generación

El estudio de composición y determinación de ppc se realizó en una zona limpia y techada propiedad de la Municipalidad de Guácimo, ubicada a 300 metros del Palacio Municipal. A cada bolsa de basura se le colocó una identificación que contenía la siguiente información: estrato socioeconómico y código de la vivienda de donde procedía. Posteriormente se pesó cada bolsa de RS, agrupando los datos individuales según el código de la vivienda en una hoja de cálculo. 
Cuadro I. Distribución de los comercios según el CIIU para el cantón de Guácimo y su contribución porcentual a la muestra seleccionada.

\begin{tabular}{|c|c|c|c|}
\hline Estrato comercial & Total de comercios & \% que representa & $\begin{array}{c}\text { Número de comercios } \\
\text { muestreados }\end{array}$ \\
\hline Supermercados & 141 & 21,04 & 11 \\
\hline Comida preparada & 119 & 17,76 & 8 \\
\hline Tiendas & 110 & 16,42 & 6 \\
\hline Servicios & 83 & 12,39 & 6 \\
\hline Almacenes & 76 & 11,34 & 3 \\
\hline Otros & 61 & 9,10 & 2 \\
\hline Organizaciones & 48 & 7,16 & 2 \\
\hline Agrícola & 23 & 3,43 & 52 \\
\hline Atención Salud & 9 & 1,34 & 100 \\
\hline Total & 670 & & 5 \\
\hline
\end{tabular}

Cada bolsa se abrió y se inspeccionó; en caso de encontrarse algún residuo peligroso, se separó manualmente para evitar riesgos a la salud de los colaboradores. Los residuos de gran volumen se cortaron con tijera de podar y cuchillo de chapia, con el objetivo de realizar adecuadamente el cuarteo para la toma de muestra del estudio de composición.

Se colocaron cerca de $200 \mathrm{~kg}$ para el cuarteo de cada estrato. La muestra se mezcló con palas y una vez lista se inició con el cuarteo (Programa de Competitividad y Medio Ambiente, CYMA, 2008); los colaboradores tomaron medidas de seguridad y fueron vacunados para realizar esta labor.

En atención al reglamento se determinaron los pesos respectivos de las I I categorías de residuos sólidos, a saber: Biodegradable, Papel/Cartón, Plástico,Vidrio, Metales, Textiles, Polilaminados, Peligrosos, Eléctricos y Electrónicos, Espuma de estiren y otros residuos.

\section{Resultados y discusión}

\section{Estudio de generación}

\section{Sector viviendas}

Para cada estrato se determinó el índice de generación y la distribución porcentual de los materiales de vertido. En el cuadro 2 se pueden observar solo los resultados de los cálculos de generación de RS por habitante diario para el sector socioeconómico alto; el resto de los cálculos es idéntico, por tal razón no se adjuntan los datos parciales. Los resultados de cada estrato socioeconómico se analizaron con herramientas estadísticas para establecer su normalidad; el $p$ value utilizado en todos los casos fue de 0,05 , y en caso contrario se aplicaron las transformaciones matemáticas necesarias para alcanzar la normalización. Posteriormente, conociendo la generación per cápita por día de cada estrato, se aplicó el test de ANOVA con un 95\% de confianza, determinándose diferencias significativas en la generación de RS entre los tres estratos socioeconómicos.

Para inferir las cantidades que podrían generarse en todo el cantón de Guácimo, se utilizó la siguiente fórmula:

$1 G_{E S} \times \% P_{E S} \times P_{t}=k g$, día CG

$\mathrm{IG}_{\mathrm{ES}}=$ Índice de generación estrato socioeconómico $\% \mathrm{P}_{\mathrm{ES}}=\%$ de población del estrato socioeconómico $P_{t=}$ población del cantón de Guácimo

kg/día CG = kilogramos por día en el cantón de Guácimo

Con estos datos se proyecta una generación diaria para todo el cantón de 14.95 toneladas (cuadro 3).

Adicionalmente, utilizando las desviaciones estándar y los resultados del cuadro 3, es posible estimar las generaciones mínimas y máximas para el cantón en el año 2013 (cuadro 4). 
Cuadro 2. Índice de generación per cápita para el estrato alto.

\begin{tabular}{|c|c|c|c|}
\hline Código & Habitantes/ vivienda & Peso total en kg & kg/persona/día \\
\hline EA-0I & 5 & 30,90 & 0,88 \\
\hline EA-02 & 3 & 19,66 & 0,94 \\
\hline EA-03 & 5 & 19,52 & 0,56 \\
\hline EA-04 & 4 & 12,60 & 0,45 \\
\hline EA-05 & 5 & 25,36 & 0,72 \\
\hline EA-06 & 3 & 17,32 & 0,82 \\
\hline EA-07 & 5 & 33,98 & 0,97 \\
\hline EA-08 & I & 6,58 & 0,94 \\
\hline EA-09 & 5 & 29,70 & 0,85 \\
\hline EA-10 & 5 & 30,30 & 0,87 \\
\hline EA-II & 4 & 26,80 & 0,96 \\
\hline EA-12 & 5 & 24,76 & 0,71 \\
\hline EA-13 & 4 & 23,98 & 0,86 \\
\hline EA-14 & 4 & 18,04 & 0,64 \\
\hline EA- 15 & 4 & 18,60 & 0,66 \\
\hline EA-16 & 4 & 12,62 & 0,45 \\
\hline EA-17 & 5 & 29,60 & 0,85 \\
\hline EA-18 & 3 & 10,32 & 0,49 \\
\hline EA-19 & 4 & 17,28 & 0,62 \\
\hline EA-20 & 2 & 13,66 & 0,98 \\
\hline EA-2I & 4 & 15,86 & 0,57 \\
\hline EA-22 & I & 6,04 & 0,86 \\
\hline EA-23 & 5 & 20,22 & 0,58 \\
\hline EA-24 & 4 & 19,90 & 0,71 \\
\hline EA-25 & 6 & 17,94 & 0,43 \\
\hline EA-26 & 5 & 19,32 & 0,55 \\
\hline EA-27 & 3 & 14,62 & 0,70 \\
\hline
\end{tabular}

Cuadro 3. Índices de generación por habitante determinados para cada estrato socioeconómico y estimación de la cantidad generada diariamente en el área de estudio.

\begin{tabular}{|c|c|c|}
\hline Estrato & Generación per cápita (kg/día/habitante) & kg/día/estrato \\
\hline Alto & $0,73 \pm 0,2$ & 2,943 \\
\hline Medio & $0,61 \pm 0,08$ & 6,485 \\
\hline Bajo & $0,44 \pm 0,09$ & 5,519 \\
\hline Totales & $0,55 \pm 0,1$ & 14,947 \\
\hline
\end{tabular}


Con fines únicamente de cumplir con lo solicitado en el Reglamento de estudios de composición, también se determinó la generación per cápita promedio del cantón en 0,55 $\pm 0,1 \mathrm{~kg} /$ día/habitante, sin embargo, debido a los resultados de esta investigación, no es posible utilizar este dato para inferir resultados posteriores.

Utilizando estos datos, y considerando los aumentos previstos de población, es posible inferir los requerimientos de camiones, el aumento de rutas, la capacidad de vertido en el relleno sanitario, las contrataciones de personal y demás factores, a fin de lograr una mayor cobertura de recolección, lo cual se abordará en un fututo artículo.

\section{Sector comercial}

Al aplicar la prueba $F$ de Fisher al grupo de comercios y el subgrupo de supermercados, no se observaron diferencias significativas en los índices de generación, por lo que se decidió unificar todos los resultados para obtener el índice de generación en todo el sector comercial. Sin embargo, las composiciones de materiales sí son muy diferentes, por lo que es necesario un análisis por separado para los tipos de comercios a fin de planificar la valorización de los RS.

Del análisis estadístico se determinó una generación del sector comercial en $7,39 \mathrm{~kg} / \mathrm{d}$ áa/comercio, con una desviación estándar de 2,72.

Aplicando los anteriores indicadores y conociendo el número de comercios se pueden deducir las generaciones máximas y mínimas de RS comerciales, según se indica en el cuadro 5.

La suma de las generaciones en el sector domiciliar y comercial ronda las 19,9 toneladas/día, con máximos y mínimos como se indican en el cuadro 6.

Con el fin de proveer un resultado de fácil manejo para la Municipalidad, se calculó el índice de generación en el cantón sumando las contribuciones de los sectores comerciales y del sector vivienda, y dividiendo este resultado entre la población total, dando una generación de 0,74 \pm 0,14kg/persona/día.

Cuadro 4. Estimación de las cantidades generadas en el área de estudio para cada estrato socioeconómico, considerando su relativa composición porcentual y el índice de generación determinado en el estudio de composición de materiales.

\begin{tabular}{|c|c|c|}
\hline Estrato & Generación mínima en toneladas/día & Generación máxima en toneladas/día \\
\hline Alto & 2,23 & 3,66 \\
\hline Medio & 5,58 & 7,39 \\
\hline Bajo & 4,34 & 6,69 \\
\hline Totales & 12,16 & 17,74 \\
\hline
\end{tabular}

Cuadro 5. Generación total de residuos por comercio en el área de estudio.

\begin{tabular}{|l|c|}
\hline Máxima cantidad en toneladas de RS comerciales/día para Guácimo & 3,13 \\
\hline Mínima cantidad en toneladas de RS comerciales/día para Guácimo. & 6,77 \\
\hline
\end{tabular}

Cuadro 6. Generación total de residuos sólidos en el área de estudio.

\begin{tabular}{|c|c|}
\hline Promedio cantidad en toneladas de RS/día & 19,90 \\
\hline Mínima cantidad en toneladas de RS/día para Guácimo & 15,29 \\
\hline Máxima cantidad en toneladas de RS/día para Guácimo & 24,52 \\
\hline
\end{tabular}


El Reglamento de estudios de composición realiza cálculos sin considerar la variabilidad de los datos. En el caso de haber utilizado el método propuesto en el Reglamento, el resultado para el índice de generación hubiese sido de 0,55 kg/persona/día para el sector viviendas y de 0,16 kg/persona/día para el sector comercios, para un total de $0,7 \mathrm{l} \mathrm{kg} /$ persona día.

El resultado calculado mediante el uso de herramientas estadísticas permite medir la incertidumbre de los resultados y planificar con base en este valor. En atención a lo recomendado por el Reglamento, se trabaja con un número absoluto que determina un valor menor al esperado, lo que podría tener efectos negativos en la recolección.

Según estos datos, se puede inferir para todo el cantón de Guácimo una generación esperada de | | | 46 toneladas de residuos al año, de las cuales solo se están depositando en el botadero 7600 toneladas al año; posiblemente el resto se esté vertiendo en sitios no autorizados, lo que implica un tremendo impacto ambiental para el cantón.

En atención a los resultados del estudio de composición y considerando que la Municipalidad de Guácimo solo cuenta con dos camiones recolectores con capacidad de 9 y 12 Toneladas, y generalmente les resulta complicado realizar varios viajes en un mismo día, son comprensibles las dificultades técnicas para cumplir, con las necesidades sanitarias del cantón, incluso en momentos de mínima recolección.

Por esta razón, los autores consideran que para un municipio pequeño como el de Guácimo podría ser conveniente contratar el servicio de recolección, con el fin de evitar los trastornos de rutas, camiones en mal estado, control y fiscalización de choferes y recolectores, incumplimiento de horarios, etc., de manera que la Municipalidad pueda enfocarse en la fiscalización.

Además las personas, por su misma concientización, ya separan los residuos, por lo que se podría aprovechar y contratar un servicio de recolección en la fuente, aprovechando los residuos valorizables, que rondan un $35 \%$, o bien establecer campañas de composteo para evitar el transporte de un 45\% de los residuos biodegradables.
En el caso del sector comercial, resulta atractiva desde el punto de vista económico la recuperación de un $65 \%$ de valorizables.

Estudio de composición de los residuos sólidos

\section{Sector vivienda}

En el cuadro 7 se muestran los resultados del estudio de composición para las viviendas; los residuos biodegradables son los de mayor generación, llegando incluso al 52\% en el caso del estrato alto.

En los tres estratos socioeconómicos se encontraron residuos peligrosos, que no se pueden desechar junto con la basura tradicional porque lo prohíbe la Ley 8839, sobre Gestión de Residuos Sólidos.

La espuma de estireno, que no se puede reciclar, solo se encontró en el estrato alto.

Para determinar la contribución porcentual de toda la población se consideró la siguiente fórmula:

$$
\mathrm{IG}_{\mathrm{ES}} \times \% \mathrm{~T}_{\text {MAT }} \% \mathrm{P}_{\mathrm{ES}} \times \mathrm{Pt}=\% \mathrm{~T}
$$

$\mathrm{IG}_{\mathrm{ES}}=$ índice de generación estrato socioeconómico

$\mathrm{T}_{\text {MAT }}=\%$ de tipo de material

$\% \mathrm{P}_{\mathrm{ES}}=\%$ de población del estrato socioeconómico

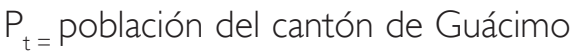

\%T: porcentaje de cada material según estrato

Se sumaron las contribuciones de cada tipo de material, por ejemplo, biodegradable, para cada estrato social y el total en $\mathrm{kg}$ se utilizó para construir la figura 4, donde se puede observar un resumen general para el área de estudio.

Con base en estos resultados, solo para el sector viviendas se prevé la posibilidad de recuperar un máximo de 5 toneladas diarias de residuos valorizables; por supuesto, este número resulta muy inferior en la operación de un centro de valorización de materiales, ya que estos vienen mezclados o en mal estado, por ejemplo, los papeles y cartones impregnados con aceites. Por lo que es urgente trabajar en la educación ambiental para acondicionar estos materiales desde la fuente, como se ha hecho en otras partes del país, además de ofrecer la recogida selectiva de los materiales.

Otro dato que llama la atención es el elevado porcentaje de residuos orgánicos (6,8 toneladas diarias). El costo de disponer estos materiales en un relleno 
Cuadro 7.Tipos de materiales encontrados en los residuos sólidos, clasificados según el estrato socioeconómico.

\begin{tabular}{|c|c|c|c|}
\hline & \multicolumn{3}{|c|}{ \% por estrato socioeconómico } \\
\hline Tipo de material & Alto & 44 & 44 \\
\hline Biodegradable & 52 & 5 & 16 \\
\hline Papel/Cartón & 6 & 12 & 13 \\
\hline Plástico & 14 & 1 & 2 \\
\hline Vidrio & 3 & 1 & 3 \\
\hline Metales & 2 & 13 & 1 \\
\hline Textiles & 5 & 0.1 & 1 \\
\hline Polilaminados & 1 & 0.07 & 2 \\
\hline Peligrosos & 2 & 2 & 0 \\
\hline Electrónicos & 0.5 & 0 & 16 \\
\hline Espuma de estireno & 0.5 & 22 & 100 \\
\hline Otros & 14 & 100 & 100 \\
\hline Total & & 13 & 2 \\
\hline
\end{tabular}

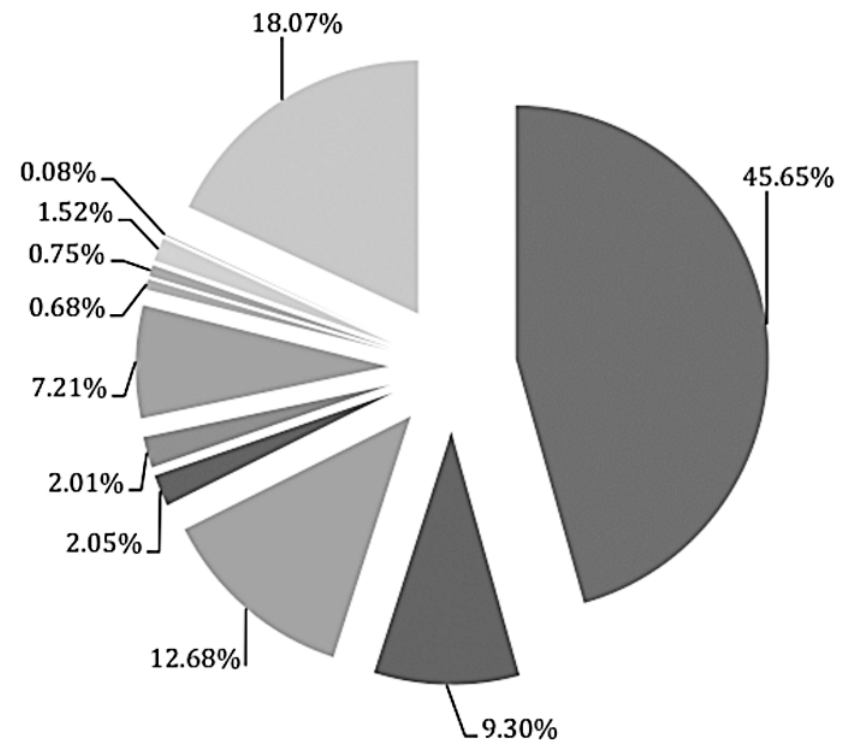

\section{-Biodegradable \\ -Papel/Cartón \\ -Plástico \\ घidrio \\ Metales \\ घTextiles \\ - Tetrabrik \\ - Peligrosos \\ Electrónicos \\ Espuma de estiren \\ - Otros}

Figura 4. Composición de los residuos sólidos en las viviendas del área de estudio.

sanitario ronda los US\$20/tonelada; en un año el municipio tendría que disponer de unos US\$50 000 más los costos de transporte, que generalmente son el triple de los costos del vertido, ascendiendo a un total de US\$200 000 anuales. Si se considera que Guácimo es un cantón donde las viviendas generalmente disponen de amplias zonas verdes, resulta importante incursionar en campañas de educación ambiental para reducir este porcentaje de material que se lleva a vertido, de manera que sea tratado en los hogares. En el país también existen empresas verdes, que ofrecen composteras para instalar en viviendas o urbanizaciones, por lo que el municipio podría planificar en sus compras la adquisición de 
estas tecnologías, en lugar de seguir invirtiendo en transporte, disposición y generación de pasivos ambientales.

\section{Sector comercial}

Se notan diferencias significativas en los porcentajes de los materiales que se desechan en los supermercados y la subcategoría otros. La diferencia porcentual entre los residuos biodegradables para ambas subcategorías ronda el 34,20\%. Una situación similar se observa con las contribuciones porcentuales de papel y cartón, con un 12,75\% en los supermercados y un 32,03\% en el subsector otros (figuras 5 y 6 ).
En el sector de supermercados el 44\% del material se puede valorizar y el $44 \%$ es biodegradable, por lo que solamente el $12 \%$ debería ir a disposición final. En el caso del subsector otros, un 68,5\% se puede valorizar y el $10 \%$ es biodegradable, por lo que el material de disposición final asciende al 21,5\%.

Se sabe por experiencias previas que es posible recuperar un porcentaje del orden del 60-80\% de materiales valorizables, después de las respectivas campañas de educación ambiental, por lo que se podría inferir que el municipio podría recuperar cerca de 4458 toneladas de materiales al año.

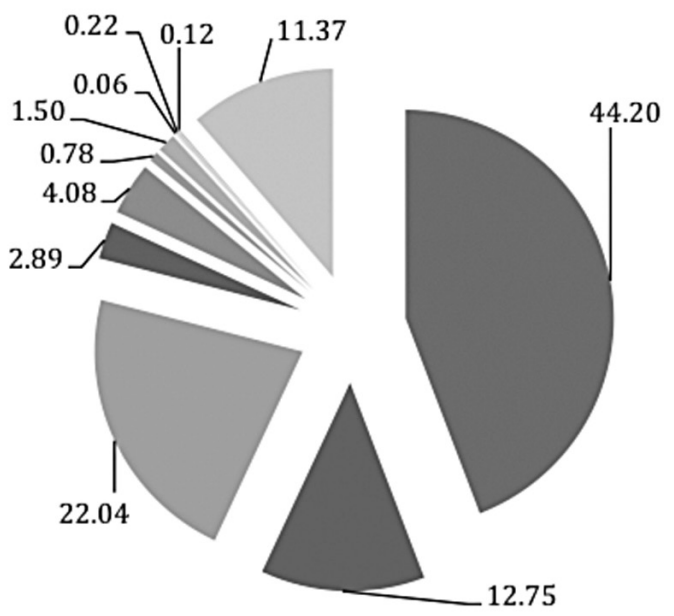

- Biodegradable

- Papel/Cartón

n Plástico

a Vidrio

Metales

- Textiles

Eolilaminados

Peligrosos

Electrónicos

Estereofón

Otros

Figura 5. Composición de los residuos sólidos en los supermercados.

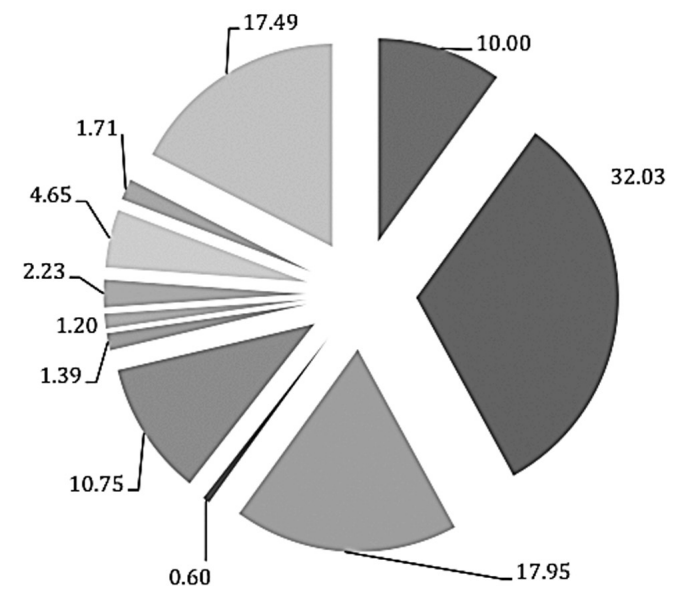

\footnotetext{
- Biodegradable

- Papel/Cartón

- Plástico

a Vidrio

$\square$ Metales

$\square$ Textiles

$\square$ Polilaminados

- Peligrosos

घ Electrónicos

घ Estereofón

a Otros
}

Figura 6. Composición de los residuos sólidos en los comercios. 


\section{Conclusiones}

En Costa Rica es obligatorio para todos los municipios contar con estudios de composición de residuos sólidos, que son una herramienta muy valiosa para la planificación de la Gestión Integral de Residuos Sólidos (GIRS). Desafortunadamente, dichos estudios se están utilizando únicamente para cumplir con la legislación nacional y no para diseñar políticas de recuperación en la fuente, educación ambiental, valorización y estrategias para el transporte y disposición.

Por esta razón es urgente apoyar al sector municipal en aspectos técnicos, administrativos y económicos, para aprovechar los resultados de los estudios de composición e índices de generación. En el caso del cantón de Guácimo, donde solo se recolecta el $65 \%$ de los RS, aunado al vertido en un botadero a cielo abierto, problemas de recolección, basuras en las calles y aceras y aparición de enfermedades asociadas a los vectores típicos de los botaderos, es estratégico utilizar esta información para establecer prioridades, contrataciones y planificar la gestión integral de los residuos. En este artículo se muestran los resultados esperados para la generación y la composición de los RS en el cantón de Guácimo. A diferencia de otros estudios, el porcentaje de materiales biodegradables disminuye con el ingreso socioeconómico. El sector alto del cantón de Guácimo desecha grandes cantidades de productos alimentarios que son propios de la capital, por ejemplo, empaques de comida rápida, alimentos procesados, etc., muchas veces en perfecto estado, lo que muestra un claro despilfarro de recursos y evidencia la brecha social que el país ha experimentado en los últimos años. Por su parte, los sectores marginales presentaron índices de generación inferiores, con un detalle que es notable destacar: una ejemplar separación en la fuente.

Por todo lo anterior, las campañas de educación ambiental, en el caso de la Municipalidad de Guácimo, deberían enfocarse en el sector alto, claro está, sin dejar de lado los otros sectores ya que se sabe que la educación ambiental es un proceso integral. Es posible proyectar a futuro los aumentos sostenidos en generación utilizando proyecciones del INEC, sin embargo, los autores consideran que es más necesario establecer pautas concretas para resolver el problema asociado a los RS que actualmente tiene la Municipalidad.
Como se pudo comprobar, es imposible en términos prácticos recoger los RS del cantón utilizando la flotilla de camiones de que dispone la Municipalidad, sumado a otros problemas de personal, disciplina, incentivos y riesgos en seguridad laboral. Por lo que se recomienda establecer carteles de licitación para la contratación del servicio, y de una vez, con recogida selectiva, aprovechando la educación ambiental que muestran algunos sectores del cantón. Dada la experiencia de otras municipalidades del país, estos carteles deben confeccionarse con topes máximos, ya que existe una cultura del sector privado de hacer grandes negocios con el gobierno. A manera de referencia, se pueden utilizar datos de otras municipalidades que ya tienen sistemas de recogida selectiva contratada. Este instrumento le permite al sector municipal centrarse en aspectos de fiscalización, en lugar de estar día a día lidiando con temas de operación, que se complican aún más por la dificultad de contratación y compra de insumos.

Obviamente, es urgente revisar la tarifa de recolección, disposición y tratamiento, simplificarla y aumentarla, de manera que permita cumplir con los requerimientos de una recolección selectiva y una disposición apropiada.

La GIR implica, entre otros, conocer con detalle el estado actual de la problemática para así poder planificar a largo plazo y brindar medidas que permitan la puesta en marcha de políticas públicas sostenibles. El primer paso para resolver este problema implica un manejo sostenible de los recursos, y partiendo de este punto se pueden comenzar a planificar acciones concretas de recuperación en la fuente. Algunos cantones del país tienen experiencias exitosas en este tema, logrando un acondicionamiento de materiales desde la fuente que es ejemplar. Adicionalmente, la valorización de los RS brinda opciones de trabajo, siempre y cuando se considere trabajar con grandes volúmenes de materiales y se respeten las garantías laborales de los trabajadores.

El contar con datos estadísticos permite la comparación y realización de proyecciones certeras con rangos de confianza para la toma de decisiones, de forma tal que las inferencias que se hacen tengan un grado de probabilidad conocido de que se den.

En lo que respecta al estrato comercial, si bien es cierto se determinó la inexistencia de diferencias significativas en el índice de generación entre los supermercados y los demás comercios, la 
composición de los residuos si varía considerablemente, por lo que a la hora de establecer estrategias de separación, recolección selectiva, programas de reciclaje o de disposición final, se debe tener claridad sobre los tipos de comercio a los que se les brinda el servicio.

\section{Agradecimientos}

Se agradece a la Vicerrectoría de Investigación y Extensión del Instituto Tecnológico de Costa Rica, por el financiamiento del proyecto "Fortalecimiento de las actividades ambientales municipales mediante el apoyo en la planificación de la gestión integral de los residuos sólidos en el Cantón de Guácimo", Código 540|-|43|-300|.

\section{Bibliografía}

Abubacar, l., Acosta, J. \& Yeomans, J. (2008). Gestión administrativa para un programa de manejo de desechos en comunidades rurales. Tierra Tropical, 4(I), 83- I I 8.

Camacho, J., Murillo, J. \& Yeomans, J. (2008). Plan de negocios para la implementación de un centro de recuperación de materiales en Guácimo, Costa Rica. Tierra Tropical, 4( I), 119-162.

Campos, R. \& Soto, S. (20|4). Análisis de la situación del estado de la GIR en el Cantón de Guácimo, Costa Rica. Tecnología en Marcha, 27(I), I|4-124.

Dicent, Y., Yeomans, J. \& Arguedas, M. (2008). Modelo de manejo de desechos sólidos ordinarios para el distrito de Pocora en Costa Rica. Tierra Tropical, 4( I), 3 I-82.

INEC. (23 de febrero, 20I I). INEC Costa Rica. Recuperado de http://www.inec.go.cr/Web/Home/GeneradorPagina.aspx

PNUD, FOMUDE. (2009). Plan de Desarrollo Humano, Guácimo. Costa Rica.

Programa Competitividad y Medio Ambiente (CYMA). (2008). Manual para la Elaboración de Planes Municipales de Gestión Integral de Residuos Sólidos. San José. 